\title{
Translator's Subjectivity from the Perspective of Eco-Translatology A Case Study of Liu Bingshan's and GAO Jian's Translations of Essays of Elia \\ Du Bei
}

\author{
School of Foreign Studies, Xi'an University, 710065
}

\author{
Keywords: Eco-translatology; Translator; Subjectivity
}

\begin{abstract}
Translator is the executor in translation, and should be given adequate attention in translating the traditional monograph on translation and static text. The traditional translation theory often thinks that the translation should be based on the original intention of the text, but with the deep understanding of eco-translatology, people found that the translation is a very spiritual work. In the process of ecological translation, the translator generally present the original meaning with target language as much as possible to conform to the internal eco-structure of the original text. Therefore, the translator tries to make the results in keeping with the habit and context of the target language in translation. Translator's subjectivity should be able to adapt to the new target language environment in a diversified language environment and make the translation meet the practical needs during the changing of the translator's marginal identity. In this paper, two different versions of the translation of Essays of Elia were analyzed, and it aimed to make more translators clarify their main role and provide a more advanced theoretical basis for the development of future translation.
\end{abstract}

\section{Introduction}

The development of translator's subjectivity in the perspective of eco-translatology is the embodiment of the transformation of social civilization, that is to say, in order to promote the communication and exchange of human society, ecological dimension has entered into a lot of social areas in the transition from industrial civilization to ecological civilization, and also has got into the study of translation. After the fusion and change of the concept of modern translatology and modern philosophy, it has been found that the work of translating language can be taken into account from the perspective of ecology translation and the important criteria for measuring translation results can be based on ecological standards. Therefore, it is inevitable for translator to grasp the translation results on this basis. The translator's subjectivity is reasonable in the process of translation, and if we want to realize its reality, we must make the subjectivity in the process of real translation.

\section{Translator's Subjectivity in the Ecological Translation Environment}

The translator's subjectivity is actually a relatively new concept. In the traditional translation theory, it has always been believed that the article is the center of translation, which requires the translator to be very objective to copy the original intention of translation texts. That is to say, translation is no spiritual, similar to the work of the tool, with the translator hidden their own personality, and the translation level embodied in the main content of the translation works. However, with the transformation of translation studies gradually from the cultural field to the ecological field in 1990s, many new theories of translation were born, such as, Skopos theory, Elaboration theory, feedback mechanism, translation manipulation school and so on. After they are integrated together, it is found that translation is actually with its own personality, and the translation results will inevitably be affected by translator in the translation process.

Definition of Ecological Translation Environment. The translation often contains a lot of the principles of ecology, so more and more attentions were paid on the impact of current global translation work on the ecology. Eco-translatology has led to three-dimensional conversion method, 
which includes language dimension, cultural dimension and communication dimension. The dimensions are applied to different translation ecosystems, and different translation strategies can be considered for effective translation. This process is based on the growing emphasis on the ecological field, ecological criticism theory, environmental language theory, language evolution ecology theory, which are the basis for the construction of the ecological translation environment, and its ideological development background. Researches on translator's subjectivity in the field of ecological environment, are in line with the traditional Chinese humanism and the doctrine of the mind. It is put forward from the perspective of a kind of natural perception and ecological translation concept, and in line with the trend of modern translation research direction.

Research Essentials of Translator's Subjectivity. In the study of the ecological translation environment, the translator is no longer in a passive position. For study on this subjective status, the ecological translation environment of the translator should be figured out, the role of translation work in multi-dimensional environment of language and text should be clarified, and the impact and constraints of the ecological translation mechanism should be learned. In order to study the subjectivity of translation from the perspective of integration and fitness, it is necessary to study the adaptability of translation content from multiple dimensions in the process of reconstructing the translated text. In the process of subjective research of translation, the nature of the research should be classified. On the basis of classification, it is necessary to study the standard of translation. Different translation texts and styles will use different translation skills, and the translator's subjectivity need to be associated with the above factors. The translator is an intelligent language conversion center. The different translation culture needs to combine with the different translation subjectivity of the original text. And we also need to understand the translator's habit, his own experiences, his most frequently

used translation skills and strategies. Therefore, the subjective role of translation will be a wide range of different effects.

Combination of Ecological Translation Environment and Translator's Subjectivity. The combination of the ecological translation environment and the translator's subjectivity requires no longer concealing the subjectivity of the translator blindly, and advocates to show the translator's personality and subjectivity as much as possible, respecting the reasonable judgment on the different translation text and translation environment transformation, and allowing the translation activities under the conditions of reasonable subjectivity for the translation works of artistic sublimation. In modern translation mechanics, people pay great attention to the theory of translation studies from the perspective of ecology. In other words, we should combine the eco-translatology with the translator's ontology to re-examine the content and adaptation of the translation insight, and think about how can the translation be able to produce more and more valuable research results by borrowing the methods and concepts of ecology. Translation is actually a process of one language and theory adapting to another language environment. From the practical point of view, we can use Survival of the Fittest from ecological translation theory to develop the eco-translatology from the extension of the concept. The combination of Evolutionary theory and translation studies makes translation a creative activity. In order to define the key concept of ecological translation and to understand the research direction of ecological translation, we can rethink the field of translation in the field of eco-translatology.

\section{The Transformation of Translator's Subjectivity in the Context of Ecological Translation}

Under the environment of ecological translation, the subjectivity of translation implicates the future development direction of translation research, which is the changing demand of the translation research object. To achieve the ability of language translation and adaptation, the translator needs to know the environment of language use, combines with various development factors in the environment, draws support from their own coordination and balance, and introduces the popular cultural and symbolic concepts. As a subjectivity of translation, the translator learns and constantly understands the combination of various cultural and practical factors in each translation process, draws the conclusion of subjectivity study, combines the comprehensive application theory of 
translation, adopts the choice of translating strategies, understands the translation behavior and the quality of alternative text, and transforms the thinking mood and the translation expression.

Translator's Marginalization and Translator's Subjectivity in the Field of Ecological Translation. Eastern and Western translation activities have a long history. From thousands of years ago, people's demand for language communication has been the beginning of the formal translation of the theoretical development of about 2000 years. In a long period of time, the translator is used as a translation tool, similar to the status of a craftsman, holding meager wages, in a marginalized situation. This kind of marginalization of the translator is contrary to the original intention of the ecological translation, which takes the translation of the article as the center, pays attention to the conscious thinking as the mainstream of the current translation, knows the translation as the service tool of politics, the ruling class, and the economic development. All of these make the translator's status clearly marginalized. Under the perspective of modern ecological translation, the marginalized situation exists till now, and is associated with the nature of the translator itself, but it has a new connotation.

Translator's Center Features in Translation of Essays of Elia under a New Era. In the process of translation, the translator should make the analysis of the preexistence structure of language and culture, convert the subjectivity and individual

-ity between the original choice and the translator's style. For translation method of Essays of Elia, under the influence of different subjective factors, translations will show a very obvious difference. When translating the text, the two translators, Liu Bingshan and Gao Jian, both appreciated and understood the Lamb's prose with the narrative style, which can handle complicated matters with ease and narrate tactfully and humbly. They can read and feel the life, so that their thoughts can deeply sink into the article. The content of Essays of Elia varied, almost covering the Lamb's all fun by stealing a little leisure from the rush, life experiences and insights. Two different translators believe that the translator's preexistence culture intertwine with the various factors into a complex network, and this complex network, extending from the author's own creative structure, is bound to cause a certain stage of the translator resonance in the process of translation. Liu Bingshan and Gao Jian, although as translators of the same period, engaged in the same prose translation with two different background knowledge. The translator's subjectivity has different influences for the choice of humanity style and the artistic conception of original translation.

TRANSFORMATION of Translator's Responsibility in Translator Centered Versions of Essays of Elia. Although Gao and Liu both felt the author's style temperament and artistic beauty in the process of translation, Liu Bingshan for the original has a very strong emotional resonance, while Gao Jian re-translated it because of the disagreement with the style of the previous translator. Each translator has his own different translation styles. In the course of the construction of the language and the transformation of speech act, Liu Bingshan hoped to reproduce the unique style of the Lamb style by combining the classical Chinese with Vernacular together, in order to experience the witty places of Lamb's prose itself as much as possible. In the process of translation, Gao Jian believes that the translation of the text should more emphasize on the impact of mental factors, that is, the translator should like their own translation firstly, and experience the literature which is in the spiritual connotation of the author's style. The most important thing is to convey the spiritual sense of the continuous heritage. The above two are the significant differences in translation, due to the different status of subjectivity.

\section{Independent Role of Translator's Subjectivity in the "Diversified Adaptability" Stage}

Translation is a comprehensive behavior of culture and language, the unique product of human society and the unique behavior of the process. Therefore, translation must be combined with economic, political, cultural, ecological and other factors to comprehensive study. As the extension of the current translation science is increasingly richer, as the subject of the person, the translator should adapt to the surrounding objective environment, and take into account the translation of the recipient as the object, the environment adaptability and translation purposes. Only in this way, translation studies will expand to a more systematic direction in the field of ecological translation . 
Adaptability of Translator's Subjectivity to Original Language. Translators in the process of objective description and elaboration of their own translation process, not only to combine their own acquired training skills, even to a certain extent, but also with personal innate language talent, the translator's knowledge can not be simple. Although the translator is still a kind of auxiliary work with typical marginalized color content, but this auxiliary content can no longer deny the translation of the main body of thinking and aesthetic orientation for the translation of the inevitable impact. In other words, From the perspective of the eco-translatology, in order to make the translation more true to restore, the translator's marginalization is able to adapt to the translator's subjective status of the auxiliary situation, and the establishment of translator status has been slowly guaranteed. The subjectivity of translation, in the original language level, must be adaptive and accurate to convey the purpose of translation style. For the original style, whether it is more coherent or combined with ancient style translation, language style must show the coincidence of the original creation. For example, for the translation of New Years' Eve in the Essays of Elia, Lamb talked about the old age, that people can approach death, but also can map peace, Gao Jian adapted a lot of non-popular of the uncommon words in the translation, such as obstruction, fine small evil, etc., which requires the readers to know a certain ancient Chinese culture, but these uncommon words can be very just right to reflect the mood of Lamb.

Adaptability of the Translator's Subjectivity to the Original Culture. Translators can more accurately grasp the original cultural level after they had a profound understanding of the original text and the author's own cultural background. At the beginning of the translation, the translator may not entirely have a detailed understanding on the author's cultural level, but with the gradually deepened sense of immersion in the translation process, the translators will be able to appreciate the original author's inner feelings change, and thus adapt to the style unconsciously. For example, when translating the "Poor Relations" in Essays of Elia, Liu Bingshan fully understood the increasingly desolate sense of Lamb's writing, and translated it with more and more desolate feeling, which is so sad and desolate, also contained in humor. Self-mockery and Lamb's original originality combined very perfectly, the cultural translation style makes the language very smooth, and the translation eventually is clear and efficient.

Adaptability of Translator's Subjectivity to Target Audience. In the above, we have described the adaptability of the two translators to the readers of the translation of the Essays of Elia. Gao's translation emphasized on ancient texts, so its readers often value the translation style which is caviar to the general and limits the readership to a certain extent, as it requires the readers with higher cultural level and quality, so the audience is relatively small minority. Liu's translation is in a colloquial and relatively humorous translation style, therefore it is more popular and conducive to the spread of culture, and the adaptability of the target readers may be stronger, so Liu's translation has a broader mass base.

Translator's Responsibility on the Choice of Translated Texts in Translation of Essays of Elia. Translator's responsibility is the ultimate conscience of every moral translator. The translator, both the imitator and the transcendent of the human spirit, carries the sacred mission and, to a large extent, economic and cultural interests. Translation is a complex work, so when Liu and Gao decided to translate Essays of Elia, they invariably had made selection on the content of the translation text. Although the life of Lamb prose is far away, but because of the cultural meaning involved, the myth of Rome, the moral mind, the refined and popular tastes, to a large extent, can adapt to the advanced development of modern culture, and has a good reference. Therefore, in the process of translating Essays of Elia, the two translators combined the required style with the free translation method.

Translator's Responsibility on the Choice of Translation Strategy in Translation of Essays of Elia. In the process of translating one language and sentences into another, the translator's responsibility is in fact fulfilling the expectation of satisfying the whole text without traces. The translator's responsibility is double, and has the selection duty of translation strategy. Taking Gao's and Liu's translations of Essays of Elia for example, Gao hoped that he can accurately express the meaning of the author's charm by creating updated vocabulary in his own way after the skilled 
scrutiny to fully understand the expression of spiritual pursuit and the original intention of the original author, while in Liu's translation, he hoped that he can reduce the destruction of the original intention as much as possible. From the view of separate words, Gao's translation has a higher degree of coincidence with the original work, but from the original intention and emotional expression of whole sentences, Liu's translation is more vivid. The two translations have their own advantages, the choice and combination of translation strategies infiltrated in the transformation process of structural components of the text and speech acts.

\section{Conclusion}

In summary, the study showed that in the process of translation, it needs the translator's subjectivity and responsibility to make the target text to adapt to the translation environment more multi-dimensionally and the process of translation coding transformation. From the perspective of the eco-translatology, the full utilization of the translator's subjective role has a more important impact on the audience, moreover, different translators for different styles of translation will inherit different cultural essence of the author. It also has an indispensable guiding role in the process of training the translators.

\section{Reference}

[1] Bian Xiaoqian. Translation Study on Chinese Culture-loaded Words in the English Version of Wolf Totem from the Perspective of Translator's Subjectivity [D]. Northwest University,2016.

[2] Huang Xiaoshan. The Translator's Responsibilities from the Perspective of Eco-translatology-A Case Study of Notes in Translation of the Collected Short Stories of Chekhov by Ru Long [J]. Journal of Guangdong Polytechnic Normal University 2014,(09):96-102+141.

[3] Li Dan. Translator's Subjectivity in Light of Eco-translatology. [D]. Ningxia University,2016.

[4] Li Hanji, Yan Yixun. A Paradigm Shift of Translator's Subjectivity in Translation Studies from Philosophy View [J]. Journal of Shenyang University (Social Science Edition),2015,(05):624-628.

[5] Li Linlin, Chen Ying. A Comparative Analysis of Translators' Subjectivity under Relevance Translation Theory and Eco-translatology [J]. Journal of Liaoning Technical University (Social Science Edition),2013,(06):625-628.

[6] Li Linlin, WangMin. Study on Translator's Subjectivity from Eco-translatology Perspective [J]. Journal of Changchun Normal University (Social Science Edition),2013,(09):78-80.

[7] Liu Haiqing. Translator-centered Research in the Post-colonial Context- from the perspective of Ecological Translation Theory $[\mathrm{J}]$. Journal of Jilin Province Institute of Educational,2012,(03):132-134.

[8] Lin Yan. Translators' Subjectivity from perspective of Translation as Adaptation and Selection: Exemplified by International Publicity Translation [J]. Journal of Shenyang University (Social Science Edition), 2016,(04):488-493.

[9] Liang Shumei. Text Translation and Translators' Subjectivity from the perspective of Intertextuality [J]. Seeker,2014,(09):120-124.

[10] Tao Xiaoting, Weng Xiaobin, He Yanqun. Empirical Study on Translator

-centered Translation Teaching from the perspective of Eco-translatology [J]. Journal of Hebei Agricultural University (Agriculture and Forestry Education Edition),2014,(06):67-71.

[11] Xu Bei. Studies on Translator's Subjectivity under Eco-translatology [D]. Hubei University, 2014.

[12] Ye Yuan. C-E Translation of TCM Terms from the Perspective of Eco-translation Theory-Embodiment of Subjectivity in Translation [J]. Journal Of Wuhu Institute Of Technology,2010,(03):4-5. 